\title{
The next level of preoperative arteries computed tomography angiography
}

\author{
Mirosław Dziekiewicz', Tomasz Markiewicz ${ }^{2,3}$ \\ 'Military Institute of Medicine, Department of Vascular and Endovascular Surgery, Warszawa, Poland \\ ${ }^{2}$ Military Institute of Medicine, Department of Pathology, Warszawa, Poland \\ ${ }^{3}$ Warsaw Technical University, Warszawa, Poland
}

Correspondence to:

Mirosław Dziekiewicz, MD, PhD Department of Vascular and

Endovascular Surgery,

Military Institute of Medicine, Szaserów 128, 04-141 Warszawa, Poland,

phone: +48261816414 e-mail: dziekiewicz@wim.mil.pl

Copyright by the Author(s), 2021 Kardiol Pol. 2021;

79 (11): 1268-1270; DOI: 10.33963/KP.a2021.0136

Received:

March 24, 2021

Revision accepted: October 12, 2021

Published online: October 13, 2021

\section{INTRODUCTION}

Atherosclerosis is usually multi-bed vascular pathology [1]. Hence, treatment often involves a wide range of endovascular operations, requiring careful preoperative access planning. Thus, vessel diameter, atherosclerotic plaque presence and volume, and tortuosity are scrutinized. However, we are still seeking a precise evaluation method, especially for tortuosity. There have been numerous attempts to calculate tortuosity through the aorta distance factor and the tortuosity index [2] or the curvature and torsion of vessels even in percutaneous coronary interventions [3]. In our study, we sought to improve computed tomography angiography (CTA) data extraction using the geometrical approach and a combination of 5 factors.

\section{METHODS}

This study was approved by the local Ethical Research Committee at the Military Institute of Medicine, Warsaw, Poland. We selected preoperatively 128 subjects, aged 35-86 years, suffering from peripheral artery disease who presented with claudication symptoms (Rutherford classification II-VI) or aortic aneurysms, excluding patients with nonatherosclerotic arteries, advanced kidney disease, and after previous interventions on the examined arteries, such as stenting, stentgraft implantation or by-passes. Patients after abdominal surgery or radiotherapy were eligible for the study. After consideration, we selected Mixture-Modeling-Based thresholding with the Gaussian/Normal mixture modeling for analyses [4]. To initialize the artery assessment, we marked the left and right femoral artery bifurcation manually and then filtered regional maxima with a custom-made tracking algorithm (Supplementary material, Figure S1). The next images in the sequence were processed automatically [4].

To detect plaques, we applied a set of 5 factors - the lumen decrease (LDF), the circumference occupancy (COF), the convex plaque area (CPAF), and the artery direction with ellipticity (EF) mapping tortuosity.

The LDF is the ratio of the plaque-occupied to the unoccupied perimeter of the artery's plane:

$$
L D F=\frac{\text { lumen }_{\text {area }}}{\text { lumen }_{\text {area }}+\text { plaque }_{\text {area }}}
$$

Since LDF has a value similar to focal and multi-focal plaque distribution, we identified the COF, calculated as follows:

$$
C O F=\frac{p_{x}}{72}
$$

$\left(p_{x}=\right.$ number of hits per plaques in the set of directions).

The next factor - CPAF - helped to establish the plaque location and structure:

$$
C P A F=\frac{\text { convex_plaque_area }}{\text { artery_area }}
$$

(Figure 1, the upper and lower left corner).

The next factor - the artery direction - is a vector joining central points of the artery plane calculated in 3D as their linear approximation (the least squares criterion). Angle changes are detected by comparing 2 consecutive directions for 2 given segments of the artery, $t_{1}$, and $t_{2}$, and the rotation angle is defined as: 


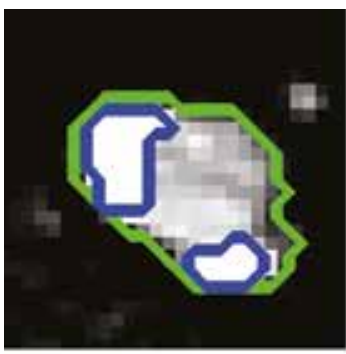

LDF factor

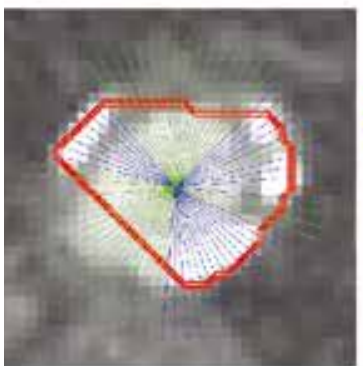

COF factor CPAF factor

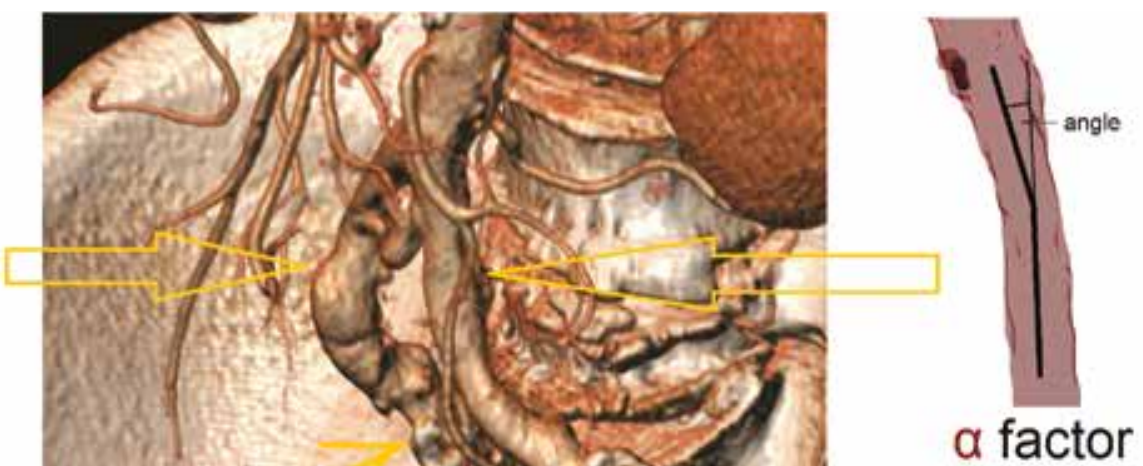

$\alpha$ factor
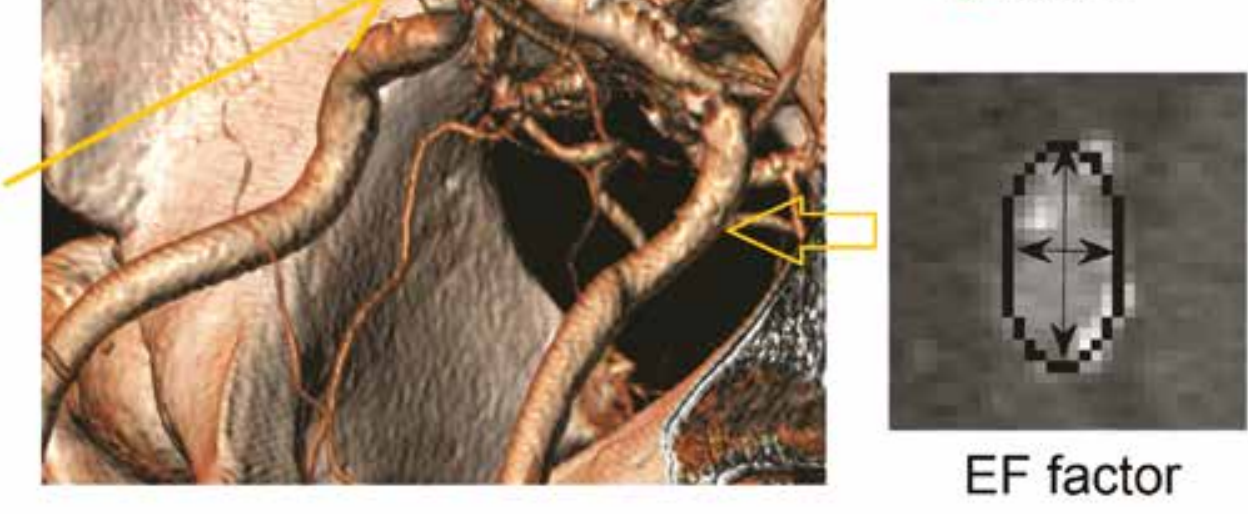

EF factor

Figure 1. Determination of LDF, COF, CPAF, $a$, and EF based on the CTA artery images. Schematic diagram of the study

Abbreviations: COF, circumference occupancy factor; CPAF, convex plaque area factor; CTA, computed tomography angiography; EF, ellipticity factor; LDF, lumen decrease factor

$$
\alpha=\arccos \left\{\frac{t_{1} \cdot t_{2}}{\left|t_{1}\right|\left|t_{2}\right|}\right\}
$$

Finally, the EF, which is the ratio of the major (marked as $2 a$ ) to the minor (marked as $2 b$ ) axes of the ellipsoidal artery plane, is calculated:

$$
E F=\frac{2 a}{2 b}
$$

In contrast to healthy arteries, the tortuous ones lack vertical planes on the CTA images, yielding high EF ratios (see Figure 1 for angle and EF measurements).

\section{Statistical analysis}

Statistical analysis was performed with the Statistica software, version 13.3 (StatSoft Polska, Kraków, Poland). The Pearson correlation coefficient and linear regression were performed to investigate the relationships between the measures (assumed significance level $=0.05$ ).

\section{RESULTS AND DISCUSSION}

Calculating the 5 aforementioned factors, we performed a successful non-invasive quantitative evaluation of the common femoral and iliac arteries from a CTA scan for the whole length of the artery (Supplementary material, Figure S2A). Except for the femoral artery division region, we detected segments with high LDF - around 280 and 230 CTA (Supplementary material, Figure S2B). Increased COF was also localized in two other regions (Supplementary material, Figure S2C). However, the CPAF continuous function was more accurate in the iliac artery than the LDF and COF (i.e., between scan 250 and 210) (Supplementary material, Figure S2D). Tortuosity was estimated on the basis of predefined measurements (4), (5). The maximum local irregularities and the average direction variability for the whole artery proved clinically significant. Supplementary Figure $S 3 A-B$ shows a sample scan from a healthy subject whose average spatial artery angles were $0.0788 \mathrm{rad} .\left(4.5^{\circ}\right)$ and $0.0849 \mathrm{rad} .\left(4.9^{\circ}\right)$ for the femoral and iliac arteries, respectively. A similar evaluation of an atherosclerotic patient (Supplementary material, Figure S4, S4A-B) was $0.2366\left(13.6^{\circ}\right)$ and $0.3686\left(21.1^{\circ}\right)$ respectively. The elliptical factor values in the healthy subject were 1.13 and 1.21 for the femoral and iliac arteries respectively, whereas in the diagnosed patient -1.40 and 1.83 respectively. The tortuosity numerical evaluation focuses on different aspects of the topographical 3-D detection. Thus, the mean angle change is a measure of both the artery twist itself and its direction, whereas the ellipticity - of the artery planes. The mean angle change explains the ellipticity in about $22 \%$ of cases with the iliofemoral segment and only in $11 \%$ of cases with the common iliac segment; Pearson correlation coefficients of the mean ellipticity and the mean angle 
changes prove linear relationship $-r=0.472 ; P<0.05$ and $r=0.346 ; P<0.05$ respectively (Supplementary material, Figure $55 A-B)$. Therefore, the most common measure in $2 \mathrm{D}$ images - the "distance metric", i.e., the ratio of the actual path length to the linear distance between the curve endpoints [3], proves unreliable. De Vries et al. [4] describes 9 tortuosity estimates, but uses an index integrating the total curvature length to the path length. Also, the leading CT equipment does not offer full assessment in long segments of arteries nor precise factor analysis to interpret exclusion or inclusion criteria for intervention.

Thus, if the system diameter was determined by an artery size, LDF higher than $15 \%$ indicates the likely success of the intervention, and if it is $60 \%-85 \%$, it requires further testing. If COF and CPAF are both less than $60 \%$ or one of them is less than $30 \%$, the artery wall is fit for intervention. Otherwise, a prior percutaneous transluminal angioplasty is advised. Also, LDF lower than $60 \%$ requires this procedure and if not possible - an alternative approach or open surgery.

Quantification of the plaques needs recalculation after initial percutaneous transluminal angioplasty. Artery tortuosity should be evaluated and compared with stent graft indications. The local angle of the artery axis bend can be compared with the border value for system implantation. The elliptical factor higher than 1.5 indicates artery course issues (kinking, significant tortuosity, etc.).

In conclusion, by applying 5 geometrical factors from the patient's CT scans we have successfully estimated differences between 2 vascular beds and identified tortuosity of the iliac arteries amplified by the accumulation of calcium in the atherosclerotic plaques. We believe this approach has the potential to become an efficient and safe preoperative arterial assessment, including even carotid or coronary arteries [5] or, for example, the percutaneous mechanical circulatory support device deployment. The proper use of this new tool may make the endovascular interventions safer for patients and interventionalists, minimizing the risk of complications connected with vascular access and endoluminal navigation.

\section{Supplementary material}

Supplementary material is available at https://journals. viamedica.pl/kardiologia_polska.

\section{Article information}

Conflict of interests: None declared.

Open access: This article is available in open access under Creative Common Attribution-Non-Commercial-No Derivatives 4.0 International (CC BY-NC-ND 4.0) license, allowing to download articles and share them with others as long as they credit the authors and the publisher, but without permission to change them in any way or use them commercially. For commercial use, please contact the journal office at kardiologiapolska@ptkardio.pl.

How to cite: Dziekiewicz M, Markiewicz T. The next level of preoperative arteries computed tomography angiography. Kardiol Pol. 2021; 79(11): 1268-1270, doi: 10.33963/KP.a2021.0136.

\section{REFERENCES}

1. Firnhaber JM, Powell CS. Lower extremity peripheral artery disease: diagnosis and treatment. Am Fam Physician. 2019; 99(6): 362-369, indexed in Pubmed: 30874413.

2. Li X, Liu X, Li X, et al. Tortuosity of the superficial femoral artery and its influence on blood flow patterns and risk of atherosclerosis. Biomech Model Mechanobiol. 2019; 18(4): 883-896, doi: 10.1007/s10237-01901118-4, indexed in Pubmed: 30652210.

3. Januszek RA, Dziewierz A, SiudakZ, et al. Predictors of periprocedural complications in patients undergoing percutaneous coronary interventions within coronary artery bypass grafts. Cardiol J. 2019; 26(6): 633-644, doi: 10.5603/CJ.a2018.0044, indexed in Pubmed: 29671862.

4. de Vries EE, Pourier VEC, van Laarhoven CJ, et al. Comparability of semiautomatic tortuosity measurements in the carotid artery. Neuroradiology. 2019; 61(2): 147-153, doi: 10.1007/s00234-018-2112-3, indexed in Pubmed: 30338348.

5. Lee SE, Park HB, Xuan D, et al. Consistency of quantitative analysis of coronary computed tomography angiography. J Cardiovasc Comput Tomogr. 2019; 13(1): 48-54, doi: 10.1016/j.jcct.2018.09.012, indexed in Pubmed: 30342980. 